\title{
Efficacy of Compression Socks to Enhance Recovery in Distance Athletes
}

\author{
Lauran Fletcher ${ }^{1}$, Scot $\operatorname{Raab}^{2}$, Sonya Sanderson ${ }^{1, *}$, Luke Vargo ${ }^{1}$ \\ ${ }^{1}$ Department of Kinesiology and Physical Education, Valdosta State University, Valdosta, 31698, GA, United States \\ ${ }^{2}$ Department of Physical Therapy and Athletic Training, Northern Arizona University, Flagstaff, 86011, AZ, United States \\ *Corresponding Author: slsanderson@valdosta.edu
}

Copyright (C) 2014 Horizon Research Publishing All rights reserved.

\begin{abstract}
The aim of the present study was to examine the effect graduated compression socks had on lower leg muscle swelling and soreness after a 2 hour (hr) run at a speed eliciting a heart rate (HR) 10-15\% lower than their HR at lactate threshold established during a baseline assessment. Compression socks were worn during the run and for $8 \mathrm{hrs}$ after the completion of the run. Baseline lower extremity (LE) volumes were measured using water displacement prior, immediately post and $24 \mathrm{hrs}$ after the $2 \mathrm{hr}$ run. Muscle discomfort was measured using an algometry prior to the $2 \mathrm{hr}$ run and approximately $24 \mathrm{hrs}$ after. Compression socks decreased LE volume measurements during and after long distance running in moderately trained distance runners but insignificantly. They also limited muscle soreness, but insignificantly. The results of this study support that compression stockings fail to enhance performance.
\end{abstract}

Keywords Compression Socks, Compression Garments, Edema, Exercise

\section{Introduction}

Compression garments have been used by patients suffering from poor circulation and lower extremity edema for over 300 years [1]. The increased external compression provided by the garments enhances extrastitial fluid return to the vascular system. A persistent accumulation of extrastitial fluid in the lower extremity increases pressure on free nerve endings causing pain, swelling, and may limit standing mobility. This condition is commonly referred to as peripheral edema [1]. While compression garments have been found to decrease edema, they have not been found to conclusively enhance the body's ability to circulate blood or aid athletic performance [2]. Healthy individuals that participate in strenuous activities in prolonged upright postures may experience a similar type of edema known as Exercise Induced Edema[3]. Exercise induced edema may also produce lower extremity pain, swelling, and a subsequent decreased ability to train [3].
There are several sports clothing companies that have begun producing knee high compression garments that range in price from $\$ 40$ to $\$ 80$ [3]. Each of the sports companies make claims that their compression garments can aid athletic performance by improving circulation and blood flow, limiting exercise induced peripheral edema of the lower extremity, supplying muscles with more oxygen, enhancing lactic acid removal, or decreased muscle soreness during and post exercise [3]. Athletes from American football, soccer, rugby, and endurance athletes such as runners and cyclists, commonly use knee high compression garments [4]. These compression socks (CS) are supposed to apply graduated compression from the ankle to the knee. Their purported purpose is to reduce edema resulting in decreased muscle soreness and swelling both during and after exercise as well as improving performance and recovery. If athletes perceived that CS's can decrease recovery time, increase abilities to train strenuously and possibly improve performance, athletes will use them to gain a competitive advantage. The problem is that the research evidence is inconclusive to support these outcomes being marketed to healthy active individuals.

Recent research protocols assessed the outcome of donning CS in various methods; this randomized clinical trial will address problematic data collection issues adding reliability to the outcome of efficacy in CS use to enhance performance. A study assessing the physiological and perceptual responses of CS use during exercise compared participants wearing CS under knee high athletic socks or simply wearing knee high athletic socks. Participants served as their own controls and separated by three days completed the exercise regime in the other condition. There were no statistically significant differences reported in RPE, perceived soreness, heart rate, or performance between the groups [5]. An additional project measured blood lactate, oxygen uptake, heart rate, and muscle soreness immediately, $24 \mathrm{hrs}$, and $48 \mathrm{hrs}$ after a 40 minute run in the conditions of low compression socks (12-15 $\mathrm{mmHg})$ and high compression socks (23-32 $\mathrm{mmHg})$. No statistically significant differences were reported [6]. Assessing the ability of CS to reduce edema, it's been recorded that socks 
with minimal compression values of $11 \mathrm{mmHg}$ could reduce evening edema in non-athletic individuals [7]. Creatine kinase $(\mathrm{CK})$ is a blood marker that can indicate muscle breakdown. Measuring CK at 1, 24, 48, 72, and $96 \mathrm{hrs}$ following a plyometric jump routine to induce muscle damage found no difference in subjects that donned a full leg compression sleeve for $12 \mathrm{hrs}$ compared to subjects not donning the compression sleeve [8]. As indicated from the research concerning the use of compression stockings as a tool to enhance performance measures or improve recovery rates, the claims of advertisers are inconclusive. The present study seeks to investigate the use of CSs as a means to enhance performance during long distance running, and investigate the effects they might have on post exercise soreness and lower extremity swelling.

\section{Materials and Methods}

\subsection{Participants}

Sixteen participants (11 males: 5 females) with a mean age of $30.5 \pm 8.7$ years participated in the study. All participants were local runners and triathletes from Valdosta, Georgia. Participants were recruited from local practices and events, as well as by word of mouth. Individuals that expressed an interest in the study were provided details via personal correspondence or e-mail. Once confirmed that a participant would take part in the study they received a copy of the IRB informed consent for review prior to reporting to the Human Performance Lab (HPL).

\subsection{Methods}

Participants completed a medical health questionnaire to assess potential risks pertaining to a $\mathrm{VO} 2$ test to volitional cessation prior to assessments. The baseline anthropometric measures of height and weight were recorded along with their self-predicted 5K race time. Participants then donned heart rate monitors, which were properly fitted for their chest size. The heart monitor was worn during both the 5-minute warm up and throughout the assessment procedures. Participants self-selected their 5-minute warm up pace after being informed at what speed their $\mathrm{VO} 2$ assessment would start. $\mathrm{VO} 2$ assessments started at a rate equivalent to one minute per $5 \mathrm{~K}$ slower than their self-predicted $5 \mathrm{~K}$ time. After completing the warm up, participants were allowed to perform any other activities that they felt they needed to prepare them for the assessment procedure for a time of 5 minutes. During this time, they were fitted for the headgear, mouthpiece, and nose plug of the metabolic system. After receiving safety briefings on how to step on and off the treadmill, participants then began the 3-minute incremented VO2 assessment. Every three minutes the treadmill speed would increase equal to a one-minute reduction in $5 \mathrm{~K}$ time. Participants' rate of perceived exertion was recorded two and a half minutes into each stage. If at any time during the test participants indicated that they wished to stop, the testing was terminated. If the researcher noted 2 minutes of running with an RER over 1, the participant was informed that ventilatory threshold had been reached and that $2 \mathrm{hr}$ run pace could be established. At this point participants could elect to max test if there was no pre-existing reason to prevent a max test or if the participant desired, they could terminate the test. Upon completion of the testing procedure, the mouth, headpiece, and nose plug were removed and the participant completed a 5-minute cool down. After the cool down, a meeting was scheduled after a minimum of $48 \mathrm{hrs}$ rest before participants returned to the HPL for their 2-hour run.

During the second scheduled meeting, participants had height and weight recorded followed by two initial types of measurements of interest. The first was an algometric pain measurement at thirteen distinguishable landmarks identified on the legs. Points 1, 2, and 3 were centered along the Achilles at 10,15, and 20 centimeters above the initial point of the Achilles tendon at the level of the medial malleolus. The fourth spot was centered posteriorly on the calf at the level of its greatest circumference. Point 5 was located halfway between point 4 and along a horizontal line to the medial border of the tibia. Points 6 and 7 were 2.5 and 5 centimeters below point 5 respectively. Points $8,9,10$, and 11 were at a vertical line along the fibula at $10,15,20$, and 25 centimeters above the lateral malleolus. Point 12 was centered halfway along a horizontal line between point 11 and the midline of the posterior calf. Point 13 was centered halfway along a horizontal line between point 10 and the midline of the posterior calf. The algometer was pressed into the marked locations with increasing pressure until the participant indicated that they felt discomfort resulting in them saying ouch or flinching away (see figure 1). At this time the pressure was immediately released and the algometric pressure reading was recorded to the nearest pound of pressure. Higher scores or pressures indicated less pain or discomfort. The second assessment was a lower extremity (LE) volume measurement completed by water displacement. The foot and LE were placed in water to a depth that reached the most prominent part of the tibial tuberosity. Water displaced by the volume of the foot and LE was measured using a graduated cylinder and weighed in grams. At this time, participants were randomly assigned to one of two conditions for the two hr run. The experimental group donned a pair of CSs exerting 18-26 mmHg of compression or the control group donning a sock exerting less than $5 \mathrm{mmHg}$ of compression. After donning the socks participants also donned a heart rate monitor. They completed a 5-minute warm up on the treadmill similar to the method they used prior to the $\mathrm{VO} 2$ assessment. After the 5 -minute warm up they were again allowed a five minute preparation, to gather water, sports drinks, or energy sources they would typically use on a $2 \mathrm{hr}$ run. They started the two $\mathrm{hr}$ run at a heart rate $10-15 \%$ below their corresponding heart rate at an RER between 1 and 1.02. After $2 \mathrm{hrs}$, the participant removed the socks, and the volumetric measurements were completed again. The displaced water 
was weighed and recorded. The participants were weighed to determine how much weight was lost and compared to the volume of fluids consumed during the $2 \mathrm{hr}$ run. Then they were given a clean pair of identical socks to wear home for eight hrs post run. A third meeting was scheduled within a 22-26 hr period post completion of the 2-hour run. The two primary measures completed at this time were follow-up LE volume displacement and objectively perceived pain. Algometric pain measurements were repeated in the exact same fashion at this post assessment as they were during the pre-assessment. The second measurement was the LE volume measurement. Again, these were completed in exactly the same fashion as the pre-assessment.

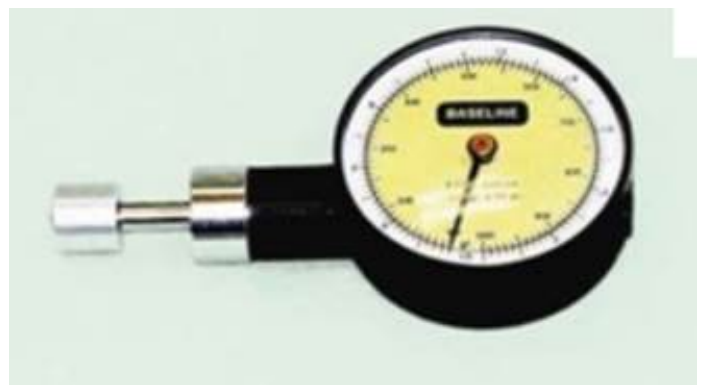

Figure 1. Algometer

\subsection{Materials}

The present study used Zoot Active Compression Socks [9]in the experimental condition to investigate the effects that CSs have on recovery and athletic performance. Zoot Active Compression Socks are comprised of $85 \%$ nylon and $15 \%$ spandex. Their graduated compression starts at $26 \mathrm{mmHg}$ at the ankle and decreases to $18 \mathrm{mmHg}$ just below the knee. Women's panty hoses were used for the control group. The panty hose were made of $88 \%$ nylon and $12 \%$ spandex which placed no measurable compression on the leg. Participants were blinded to the two different groups and the socks used during the study. Participants had been informed that they would wear a sock to measure the effect on LE fluid displacement and subjective pain.

\section{Results}

During the 2-hour run, participants consumed $1.10 \pm 0.50$ $\mathrm{L}_{2} \mathrm{O}$ and body mass decreased $0.90 \pm 0.75 \mathrm{~kg}$. A one-way ANOVA indicated no significant difference between weight loss in socked conditions $(F=1.639, d f=15, p=.221)$ or fluid consumed $(F=1.08, d f=15, p=.316)$. The mean LE volume lost immediately after exercise in the Zoot CSs was $144.9 \pm 149.17$ grams compared to $55.5 \pm 278.16$ grams in the panty hose. The mean LE volume lost after $24 \mathrm{hrs}$ in the Zoot CSs was only $3.4 \pm 135.47$ grams and the panty hose group gained $61.8 \pm 278.15$ grams of volume. However, no significant difference was found between volume measures of the LE immediately after $(F=0.859, d f=15, p=.370)$ or $24 \mathrm{hrs}(F=0.356, d f=15, p=.560)$ after exercise in either socked condition. The 13 individual algometer point values on both legs (26 total assessment points) where combined for a total LE assessment of pain. Higher initial scores indicated less pain or higher tolerance to pressure. At $24 \mathrm{hrs}$ post exercise, the group wearing pantyhose had a mean decrease in pain tolerance of $89.25 \pm 89.07$ points and the Zoot participants had a decrease in pain tolerance of $40.31 \pm$ 113.78 points. The participants donning the Zoot compression socks had a smaller increase in muscle soreness at 24 hrs but not significantly $(F=.917, d f=15, p=.354)$. The mean distance participants ran during $2 \mathrm{hrs}$ at a controlled heart rate was $12.29 \pm 1.79$ miles, participants in pantyhose ran $11.85 \pm 1.42$ miles and participants in Zoot CSs ran $12.74 \pm 2.09$ miles. Again, no significant differences existed between the conditions $(F=0.984, d f=15, p=.34)$. This study found that CSs decreased LE volume measurements, limited pain increase $24 \mathrm{hrs}$ post exercise, and allowed participants to run further without increasing heart rate. However, no statistically significant differences were found (see table 1).

Table 1. Zoot/Panty Hose ANOVA

\begin{tabular}{|c|c|c|c|c|c|c|}
\hline \multirow[b]{2}{*}{ Variable } & \multicolumn{2}{|c|}{$\underline{\text { Zoot Compression Sock }}$} & \multicolumn{2}{|c|}{$\underline{\text { Panty Hose }}$} & \multicolumn{2}{|c|}{ ANOVA } \\
\hline & $\underline{\mathrm{M}}$ & $\underline{\mathrm{SD}}$ & $\underline{\mathrm{M}}$ & $\underline{\mathrm{SD}}$ & $\underline{F}$ & $\underline{p}$ \\
\hline Weight Lost & 1.13 & .45 & .66 & .94 & 1.639 & 0.221 \\
\hline Fluid Consumed & .97 & .40 & 1.2 & .58 & 1.079 & 0.316 \\
\hline Distance Completed & 12.74 & 2.09 & 11.85 & 1.42 & 0.984 & 0.338 \\
\hline Change in Pain & 40.31 & 113.78 & 89.25 & 89.07 & 0.917 & 0.354 \\
\hline $\begin{array}{c}\text { Immediate Volume Change (LE } \\
\text { Swelling) }\end{array}$ & 144.97 & 149.17 & 55.50 & 228.64 & 0.859 & 0.370 \\
\hline $24 \mathrm{hr}$ Volume Change (LE Swelling) & 3.40 & 135.47 & -61.85 & 278.15 & 0.356 & 0.560 \\
\hline
\end{tabular}

Body weight measured in $\mathrm{Kg}$, Volume changes measured in $\mathrm{mg}$, fluids consumed measured in L, distance in miles, pain was pounds of pressure tolerated. 


\section{Discussion}

This research examined the efficacy of compression sock use by runners to see if CSs made a significant difference in recovery or performance. The Zoot brand CSs used purport a $29 \%$ decrease in lactic acid which would reduce delayed onset muscle soreness (DOMS). However, the researchers found no significant evidence that the DOMS after a $2 \mathrm{hr}$ run was diminished. Likewise, the CSs did decrease LE volume immediately after the run and $24 \mathrm{hrs}$ after the 2-hour run but not significantly. The article authored by Ali [5] measured perceived soreness, in order to examine the effects of wearing graduated compression stockings on and the perception of soreness using an 11-point scale. The current study assessed discomfort using a quantifiable measure. Both studies failed to find a statistically significant difference in pain as a result of wearing CSs. The study conducted by Ali [6] had participants run for 40 minutes. This is a time span that would cover $5 \mathrm{~K}$ and $10 \mathrm{~K}$ races and participants in these events do wear CSs. However, other athletes wear CS for much long when they compete in ultra-distance triathlons or marathons. It is conceivable that a longer exercise bout may present with different outcomes when looking at various socked conditions. This Ali study and the current one failed to find statistically significant differences in any measures assessed related to performance and recovery. Previous studies failed to blind participants to the socked condition. This may result in a placebo effect and skew perceived exertion, soreness, and might impact effort. The current study blinded participants to the socks intent and they had no knowledge of the other condition. The use of volumetric measurement also added a quantifiable measure of LE swelling while the algometer provided a less subjective assessment of discomfort. While there was a trend for participants in this study wearing Zoot CSs to have less increases in pain after exercise and a decrease in swelling, it was not significant. This may be due to the limited number of participants. Future studies should aim to increase participant numbers adding power to statistical analysis.

\section{Conclusion}

This current research fails to support the claims made by manufactures of compression garments to increase performance, decrease post exercise discomfort, or improve recovery time. If athletes feel that compression garments are worthwhile and beneficial, they can use them at their own preference. There are no indications that the compression garments reviewed and studied in these articles are detrimental to performance or recovery. There may also be a placebo effect to wearing compression socks and that is difficult to assess.

\section{REFERENCES}

[1] Palfreyman, S.J., Lochiel, R., \& Michaels, J. A. A systematic review of compression therapy for venous leg ulcers. Vascular Medicine, Vol. 3, 301-313, 1998

[2] Coulborn, A. \& Hampton, S. Understanding assessment and management of lymphedema. Nursing and Residential Care, Vol. 14, No. 10, 516-520, 2012.

[3] Bonow, R. O., Mann, D. L., Zipes, D. P., \& P. Libby. Braunwald's heart disease: A textbook of cardiovascular medicine (9 ed.). Philadelphia, PA: Saunders Elsevier, 2011.

[4] Bradley L. Venous hemodynamics and the effects of compression stockings.British Journal of Community Nursing.Vol. 6, No. 4, 165-175, 2001.

[5] Ali, A., Caine, M.P., \& Snow, B.G. Graduate compression stockings: Physiological and perceptual responses after exercise. Journal of Sports Sciences, Vol. 25, No.4, 413-419, 2006.

[6] Ali, A., Creasy, R., \& Edge, J. Physiological effects of wearing graduated compression stockings during running. European Journal of Applied Physiology, Vol. 109, No.6, 1017-1025, 2010.

[7] Partsch, H., Winiger, J., \&Lun, B. Compression stockings reduce occupational leg swelling. Dermatologic Surgery, Vol. 30, 737-743, 2004.

[8] Kraemer, W. J., French, D.N., \&Spiering, B.A. Compression in the treatment of acute muscle injuries in sport. International Sports Medicine Journal, Vol. 5, No. 3, 200-208, 2004.

[9] Zoot. Available at:http://zootsports.com/mens/accessories/m -ultra-2-crx-sock-s13, 2013. 